\title{
Retained Products of Conception as an Etiology for Endometritis
}

\author{
Pietro Bortoletto $^{1}$, Phillip Romanksi ${ }^{2}$, Nina Schatz-Siemers ${ }^{2}$, and Steven Spandorfer ${ }^{3}$ \\ ${ }^{1}$ Weill Cornell Medicine \\ ${ }^{2}$ Affiliation not available \\ ${ }^{3}$ Ronald O Perelman and Claudia Cohen Center for Reproductive Medicine
}

June 14, 2021

\section{Retained Products of Conception as an Etiology for Endometritis}

Pietro Bortoletto, MD ${ }^{1}$, Phillip A. Romanski, MD ${ }^{1}$, Nina Schatz-Siemers, DO ${ }^{2}$, Steven D. Spandorfer, MD ${ }^{1}$

1 The Ronald O. Perelman and Claudia Cohen Center for Reproductive Medicine, Weill Cornell Medical College, 1305 York Ave, 7th Floor, New York, NY 10021, USA.

2 Department of Pathology and Laboratory Medicine, Weill Cornell Medicine-New York Presbyterian Hospital, New York, NY 10021, USA.

\section{Corresponding Author:}

Pietro Bortoletto, MD

The Ronald O. Perelman and Claudia Cohen Center for Reproductive Medicine

1305 York Avenue, 6th Floor

New York, NY 10021

Phone: (646) 962-2764

Email:pietrobortolettomd@gmail.com

Running Head: Retained Products of Conception

Commentary

Chronic endometritis is a controversial and complex condition encountered by reproductive specialists. In the infertile population, the prevalence of chronic endometritis has been estimated to between $2.8-37.0 \%$ but as high as $56.8 \%$ in one study, compared to around $10 \%$ in the general population undergoing hysterectomy for benign pathology. ${ }^{1,2}$ The overwhelming prevalence of chronic endometritis in infertile women suggests that both infectious and non-infectious etiologies must be considered as causative factors. The pathologic diagnosis of chronic endometritis requires the identification of plasma cells on histologic sections of endometrial samples. Plasma cells present within the endometrium are often associated with reactive stroma, increased lymphocytic infiltrate, lymphoid aggregates and disordered glandular architecture; however, these changes are not required to render a diagnosis of chronic endometritis. ${ }^{3}$ When present, chronic endometritis has been implicated in reduced reproductive potential, primarily through decreased embryo implantation and recurrent miscarriage. Several studies have suggested that treatment with antibiotics may improve subsequent reproductive outcomes in women with chronic endometritis, making screening and diagnosis essential to improving outcomes. However, even with appropriate antibiotic therapy, cure rates for chronic endometritis are approximately $32.3 \%$ after a single course of antibiotics, which supports the hypothesis that non-infectious causes are also a common cause of this condition. ${ }^{4}$ This evolving understanding of causative factors is central 
to the complexity of managing chronic endometritis. One of the increasingly important etiologies for chronic endometritis is retained products of conception (RPOC) which can propagate a persistent inflammatory milieu within the endometrium.

Retained products of conception occur in up to $17.8 \%$ of first trimester pregnancy losses. ${ }^{5}$ For the vast majority of women, RPOC will be spontaneously expelled from the uterus without further surgical intervention. However, with expectant management the median time to sonographic resolution has been reported to be upwards of 111 days (median: 84, IQR: 50-111). ${ }^{6}$ In the infertile population, management strategies that help to expedite subsequent attempts at conception are often preferred so interventions such as misoprostol, mifepristone, and operative hysteroscopy are routinely recommended. Furthermore, prolonged retention of pregnancy tissue and blood products within the uterine cavity is not without its own risk as the miscarried tissue may act as culture media for genital tract pathogens resulting in infection and inflammation that can have deleterious reproductive consequences.

Increasingly, the concept of an impaired inflammatory state of the endometrium (IISE) has been used to describe both infectious and non-infectious etiologies that have traditionally been consolidated into the catch-all of "infectious chronic endometritis". ${ }^{7}$ Microbial pathogens, but also trauma, ischemia, and foreign bodies are potent activators of the body's inflammatory response. Depending on the nature of the inciting event, IISE can be classified as transient, repeated, or persistent. Acutely, inflammation is a consequence of activation of the innate immune system for immediate, non-specific host defense and is a transient event. However, over-activation of pro-inflammatory pathways and/or failure to transition to a post-inflammatory state may make the endometrium susceptible to repeated and eventually persistent impaired inflammatory states. This repetitive or prolonged inflammatory state may alter the microenvironment of the endometrium and increase susceptibility to pathogens leading to further inflammation - a vicious cycle.

Our group has recently reported on a series of 111 women without a history of chronic endometritis who underwent operative hysteroscopy for retained products of conception and found that nearly 1 in 4 women (23.4\%) had evidence of chronic endometritis on histopathology. ${ }^{8}$ Miscarriage, in and of itself, is an acute inflammatory event with a broad range of cytokines being released and immune cells being recruited to and developing within the endometrium. However, as previously mentioned, most women will spontaneously pass the products of conception allowing the endometrium to transition to a post-inflammatory state. When the products of conception do not pass completely, the resulting RPOC serves as a foreign body within the endometrium that may serve to stimulate a persistent inflammatory environment. Viewing chronic endometritis through the lens of a persistent or recurrent impaired inflammatory state helps to conceptualize this novel finding and may explain why a large percentage of women fail to respond to treatment with antibiotics despite appropriately targeted therapy. More importantly, it raises questions about how to optimally manage retained products of conception to prevent the damage to the endometrium from prolonged inflammatory stimuli.

In our study cohort, the median time from sonographic diagnosis of RPOC to operative hysteroscopy was 11 days (IQR: 6-20). Despite this relatively short interval to surgical management, diagnostic of chronic endometritis (i.e. presence of plasma cells) were present in some of the tissue specimens as detailed above. This raises consideration of whether passive management strategies that increase the latency between diagnosis to resolution of RPOC may prolong the inflammatory process within the endometrium. This may have the potential to cause long term deleterious reproductive outcomes and enhance susceptibility to microbial infection. While more work is needed to better understand optimal management strategies to minimize persistent inflammation, we believe hysteroscopic management is one option that affords patients an opportunity for timely and targeted resolution to retained tissue that may have downstream beneficial reproductive consequences.

Until now, management of retained products of conception has focused on evacuating the tissue either through surgical intervention or through the use of prostaglandin E1 analogues and/or anti-progestins. Reframing our understanding of chronic endometritis as a persistent impaired inflammatory state that may have noninfectious etiologies invites the possibility of novel therapeutic adjuncts beyond antibiotic therapy. Synthetic 
glucocorticoids, for example, have been used in the management of reproductive failure for their potent and broad-spectrum anti-inflammatory and immunosuppressive properties. ${ }^{9}$ It is possible that their ability to dampen inflammation within the endometrium perioperatively may be of added benefit beyond simple resection of the retained tissue. Of course, further studies are needed to better characterize their utility and safety as a percentage of patients with RPOC may also have an infectious etiology that warrants antibiotic therapy.

If we are to treat chronic endometritis effectively, it is incumbent that we reframe our understanding of the condition beyond an infectious pathogen resulting in infiltrative plasma cells within the endometrium. Allowing for both infectious and non-infectious etiologies to be causative agents in impaired inflammatory states of the endometrium (IISE) increases our ability to identify conditions or events that may have deleterious reproductive outcomes. Retained products of conception, by nature of the inflammatory and then ischemic processes preceding them, may result in recurrent and eventually persistent inflammatory damage to the endometrium. Optimal management strategies for RPOC, with a focus on timely resolution and the potential for anti-inflammatory adjuncts, require further attention and study. Only then may we stand a chance to unravel this complex and likely multifactorial condition that we still refer to singularly as "chronic endometritis".

\section{Disclosure of Interest:}

The authors have no financial conflict of interest to disclose

\section{Contribution to Authorship:}

All authors participated equally in the preparation of the commentary

\section{Details of Ethics Approval:}

Not required

\section{Funding:}

No source of funding to declare

\section{References}

1. Kasius JC, Broekmans FJM, Sie-Go DMDS, Bourgain C, Eijkemans MJC, Fauser BC, et al. The reliability of the histological diagnosis of endometritis in asymptomatic IVF cases: a multicenter observer study. Hum Reprod. 2012 Jan 1;27(1):153-8.

2. Cicinelli E, De Ziegler D, Nicoletti R, Colafiglio G, Saliani N, Resta L, et al. Chronic endometritis: correlation among hysteroscopic, histologic, and bacteriologic findings in a prospective trial with 2190 consecutive office hysteroscopies. Fertil Steril. 2008 Mar;89(3):677-84.

3. Margulies SL, Dhingra I, Flores V, Hecht JL, Fadare O, Pal L, Parkash V. The Diagnostic Criteria for Chronic Endometritis: A Survey of Pathologists. Int J Gynecol Pathol. 2020 Dec 14.

4. Cicinelli E, Matteo M, Tinelli R, Lepera A, Alfonso R, Indraccolo U, et al. Prevalence of chronic endometritis in repeated unexplained implantation failure and the IVF success rate after antibiotic therapy. Hum Reprod. 2015 Feb 1;30(2):323-30.

5. Bosch T van den, Daemen A, Schoubroeck DV, Pochet N, Moor BD, Timmerman D. Occurrence and Outcome of Residual Trophoblastic Tissue. J Ultrasound Med. 2008;27(3):357-61.

6. Wada Y, Takahashi H, Suzuki H, Ohashi M, Ogoyama M, Nagayama S, et al. Expectant management of retained products of conception following abortion: A retrospective cohort study. Eur J Obstet Gynecol Reprod Biol. 2021 May 1;260:1-5. 
7. Drizi A, Djokovic D, Laganà AS, van Herendael B. Impaired inflammatory state of the endometrium: a multifaceted approach to endometrial inflammation. Current insights and future directions. Przeglad Menopauzalny Menopause Rev. 2020 Jul;19(2):90-100.

8. Elder S, Bortoletto P, Romanski PA, Spandorfer S. Chronic endometritis in women with suspected retained products of conception and their reproductive outcomes. Am J Reprod Immunol N Y N 1989. 2021 Feb 28;e13410.

9. Achilli C, Duran-Retamal M, Saab W, Serhal P, Seshadri S. The role of immunotherapy in in vitro fertilization and recurrent pregnancy loss: a systematic review and meta-analysis. Fertil Steril. 2018 Nov;110(6):1089-100. 